\title{
Chiral Solitons with Bohm Potential by Modified Simple Equation Method and Trial Equation Scheme
}

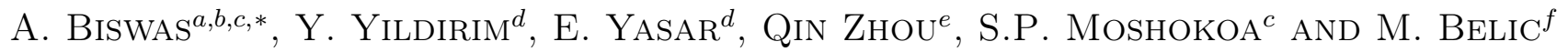 \\ ${ }^{a}$ Department of Physics, Chemistry and Mathematics, Alabama A\&M University, Normal, AL 35762-7500, USA \\ ${ }^{b}$ Department of Mathematics, King Abdulaziz University, Jeddah-21589, Saudi Arabia \\ ${ }^{c}$ Department of Mathematics and Statistics, Tshwane University of Technology, Pretoria-0008, South Africa \\ ${ }^{d}$ Department of Mathematics, Faculty of Arts and Sciences, Uludag University, 16059 Bursa, Turkey \\ ${ }^{e}$ School of Electronics and Information Engineering, Wuhan Donghu University, Wuhan-430212, \\ People's Republic of China \\ ${ }^{f}$ Institute of Physics, Belgrade, Pregrevica 118, 11080 Zemun, Serbia \\ (Received January 10, 2018; in final form December 21, 2018)
}

\begin{abstract}
In this paper we obtain soliton solutions to chiral nonlinear Schrödinger's equation with the Bohm potential by the modified simple equation method and trial equation method. Solitons and shock wave solutions are obtained. Additionally, singular periodic solutions are revealed as a by-product of these approaches and these are also listed. The existence criteria of these form of solutions are also presented.
\end{abstract}

DOI: 10.12693/APhysPolA.134.1120

PACS/topics: solitons, Bohm potential, modified simple equation method, trial equation method

\section{Introduction}

The dynamics of chiral solitons along with its perturbations has been an ongoing research area, in nuclear physics, for the past few decades [1-15]. This model has gained popularity ever since its first appearance in the literature by Jackiw et al. during 1990 [9]. Since this point onwards, this is also famously referred to as the Jackiw-Pi model. Later, this model was studied in $(2+1)$-dimensions and time-dependent coefficients [3, 4]. Subsequently, this area of research expanded along several avenues. These include soliton perturbation theory [2], the Lie symmetry analysis [12], semi-inverse variational principle [5], numerical simulation of the governing model [8] and finally the generalization of the model to retrieve solitons and other solutions [14]. This paper revisits the governing model that is chiral nonlinear Schrödinger's equation (C-NLSE) with the Bohm potential that is treated as a perturbation term. There are two integration schemes implemented to extract solitons and shock wave solutions to the perturbed model with the Bohm potential. These are simple equation method and trial equation algorithm. The existence criteria of these solutions are also presented. The details now follow.

\subsection{Governing model}

The perturbed C-NLSE with the Bohm potential is given by

$$
\mathrm{i} q_{t}+a q_{x x}+\mathrm{i} b\left(q q_{x}^{*}-q^{*} q_{x}\right) q=\mathrm{i} \alpha q \frac{|q|_{x x}}{|q|} .
$$

\footnotetext{
* corresponding author
}

In Eq. (1), $q$ is the complex valued dependent variable, $x, t$ are the independent variables. Moreover, in (1), the first term represents the evolution term which governs how the wave evolves with time, the second is the dispersion term with coefficient $a$ and the third term is through derivative coupling, its coefficient $b$ is the nonlinear coupling constant. This kind of nonlinearity is also known as the current density. The right-hand side of (1) is called the Bohm potential that is also known as the internal self-potential that was introduced by de Broglie and explored by Bohm to introduce the hidden variable theory, its coefficient is denoted by $\alpha$. Here, $a, b$ and $\alpha$ are all real-valued constants.

\section{A rapid overview of modified simple equation method} form

Suppose we have a nonlinear evolution equation in the

$$
P\left(u, u_{t}, u_{x}, u_{t t}, u_{x t}, u_{x x}, \ldots\right)=0,
$$

where $P$ is a polynomial in $u(x, t)$ and its partial derivatives in which the highest order derivatives and nonlinear terms are involved. In the following, we give the main steps of this method.

Step-1: We use the transformation

$$
u(x, t)=U(\xi), \quad \xi=x-c t,
$$

where $c$ is a constant to be determined, to reduce Eq. (2) to the following ordinary differential equation (ODE):

$$
Q\left(U, U^{\prime}, U^{\prime \prime}, U^{\prime \prime \prime}, \ldots\right)=0,
$$

where $Q$ is a polynomial in $U(\xi)$ and its total derivatives, while $^{\prime}=\mathrm{d} / \mathrm{d} \xi$. 
Step-2: We suppose that Eq. (4) has the formal solution

$$
U(\xi)=\sum_{l=0}^{N} a_{l}\left(\frac{\psi^{\prime}(\xi)}{\psi(\xi)}\right)^{l},
$$

where $a_{l}$ are constants to be determined, such that $a_{N} \neq 0$, and $\psi(\xi)$ is an unknown function to be determined later.

Step-3: We determine the positive integer $N$ in Eq. (5) by considering the homogeneous balance between the highest order derivatives and the nonlinear terms in Eq. (4).

Step-4: We substitute (5) into (4), then we calculate all the necessary derivatives $U^{\prime}, U^{\prime \prime}, \ldots$ of the unknown function $U(\xi)$ and we account the function $U(\xi)$ . As a result of this substitution, we get a polynomial of $\psi^{\prime}(\xi) / \psi(\xi)$ and its derivatives. In this polynomial, we gather all the terms of the same power of $\psi^{-j}(\xi), j=0$, $1,2, \ldots$ and its derivatives, and we equate with zero all the coefficients of this polynomial. This operation yields a system of equations which can be solved to find $a_{k}$ and $\psi(\xi)$. Consequently, we can get the exact solutions of Eq. (2).

\subsection{Application to $C$-NLSE}

In order to solve Eq. (1) by the modified simple equation method, we use the following wave transformation:

$$
q(x, t)=U(\xi) \mathrm{e}^{\mathrm{i} \phi(x, t)}, \quad q^{*}(x, t)=U(\xi) \mathrm{e}^{-\mathrm{i} \phi(x, t)},
$$

where $U(\xi)$ represents the shape of the pulse, $\xi=$ $k(x-v t)$ and $\phi(x, t)=-\kappa x+\omega t+\theta$. The function $\phi(x, t)$ is the phase component of the soliton, $\kappa$ is the soliton frequency, while $\omega$ is the wave number, $\theta$ is the phase constant and $v$ is the velocity of the soliton.

Substituting Eq. (6) into Eq. (1) and then decomposing into real and imaginary parts yields a pair of relations. The imaginary part gives

$$
(2 a \kappa+v) k U^{\prime}+\alpha k^{2} U^{\prime \prime}=0,
$$

while the real part gives

$$
a k^{2} U^{\prime \prime}-\left(a \kappa^{2}+\omega\right) U-2 b \kappa U^{3}=0 .
$$

It needs to be noted that Eqs. (7) and (8) are to be solved together in order to integrate Eq. (1). From Eq. (7), it is possible to determine the velocity of the soliton by solving for $v$ in terms of the remaining parameters, where the soliton expression for the function $U$ is given later in (9) and (24).

\subsubsection{Case-1}

Balancing $U^{\prime \prime}$ with $U^{3}$ in Eq. (8), then we get $N=1$. Consequently, we reach

$$
U(\xi)=a_{0}+a_{1}\left(\frac{\psi^{\prime}(\xi)}{\psi(\xi)}\right) .
$$

Substituting Eq. (9) into Eq. (8) and then setting the coefficients of $\psi^{-j}, j=0,1,2,3$ to zero, we obtain a set of algebraic equations as follows:

$$
\begin{aligned}
& \psi^{-3} \text { coeff.: } \\
& -2 a_{1}\left(\psi^{\prime}\right)^{3}\left(\kappa b a_{1}^{2}-a k^{2}\right)=0,
\end{aligned}
$$

$$
\begin{aligned}
& \psi^{-2} \text { coeff.: } \\
& -3 a_{1} \psi^{\prime}\left(2 \kappa b a_{0} a_{1} \psi^{\prime}+a k^{2} \psi^{\prime \prime}\right)=0, \\
& \psi^{-1} \text { coeff.: } \\
& -a_{1}\left[\left(6 \kappa b a_{0}^{2}+a \kappa^{2}+\omega\right) \psi^{\prime}-a k^{2} \psi^{\prime \prime \prime}\right]=0, \\
& \psi^{0} \text { coeff.: } \\
& -a_{0}\left(2 \kappa b a_{0}^{2}+a \kappa^{2}+\omega\right)=0 .
\end{aligned}
$$

Solving this system, we obtain

$$
\begin{aligned}
& a_{0}= \pm \sqrt{-\frac{a \kappa^{2}+\omega}{2 b \kappa}}, \\
& a_{1}= \pm \sqrt{\frac{a}{b \kappa} k}
\end{aligned}
$$

and

$$
\begin{aligned}
& \psi^{\prime \prime}= \pm \frac{1}{k} \sqrt{-\frac{2\left(a \kappa^{2}+\omega\right)}{a}} \psi^{\prime}, \\
& \psi^{\prime \prime \prime}=-\frac{2\left(a \kappa^{2}+\omega\right)}{a k^{2}} \psi^{\prime} .
\end{aligned}
$$

From Eqs.(15) and (16), we can deduce that

$$
\psi^{\prime}= \pm \sqrt{-\frac{a}{2\left(a \kappa^{2}+\omega\right)}} k c_{1} \mathrm{e}^{ \pm \frac{1}{k} \sqrt{-\frac{2\left(a \kappa^{2}+\omega\right)}{a}} \xi},
$$

and

$$
\psi=-\frac{a k^{2}}{2\left(a \kappa^{2}+\omega\right)} c_{1} \mathrm{e}^{ \pm \frac{1}{k} \sqrt{-\frac{2\left(a \kappa^{2}+\omega\right)}{a}} \xi}+c_{2},
$$

where $c_{1}$ and $c_{2}$ are constants of integration. Substituting Eq. (17) and (18) into Eq. (9), we obtain the following exact solution to Eq. (1):

$$
\begin{aligned}
& q(x, t)=\left[ \pm \sqrt{-\frac{a \kappa^{2}+\omega}{2 b \kappa}} \pm \sqrt{\frac{a}{b \kappa} k}\right. \\
& \left.\quad \times\left(\frac{ \pm \sqrt{-\frac{a}{2\left(a \kappa^{2}+\omega\right)}} k c_{1} \mathrm{e}^{ \pm \frac{1}{k} \sqrt{-\frac{2\left(a \kappa^{2}+\omega\right)}{a}} k(x-v t)}}{-\frac{a k^{2}}{2\left(a \kappa^{2}+\omega\right)} c_{1} \mathrm{e}^{ \pm \frac{1}{k}} \sqrt{-\frac{2\left(a \kappa^{2}+\omega\right)}{a}} k(x-v t)+c_{2}}\right)\right] \\
& \quad \times \mathrm{e}^{\mathrm{i}(-\kappa x+\omega t+\theta)} .
\end{aligned}
$$

If we set

$$
c_{1}=-\frac{2\left(a \kappa^{2}+\omega\right)}{a k^{2}} \mathrm{e}^{ \pm \frac{1}{k} \sqrt{-\frac{2\left(a \kappa^{2}+\omega\right)}{a}} \xi_{0}}, \quad c_{2}= \pm 1,
$$

we obtain

$$
\begin{gathered}
q(x, t)= \pm \sqrt{-\frac{a \kappa^{2}+\omega}{2 b \kappa}} \\
\times \tanh \left(\frac{1}{k} \sqrt{-\frac{a \kappa^{2}+\omega}{2 a}}\left(k(x-v t)+\xi_{0}\right)\right) \mathrm{e}^{\mathrm{i}(-\kappa x+\omega t+\theta)}, \\
q(x, t)= \pm \sqrt{-\frac{a \kappa^{2}+\omega}{2 b \kappa}} \\
\times \operatorname{coth}\left(\frac{1}{k} \sqrt{-\frac{a \kappa^{2}+\omega}{2 a}}\left(k(x-v t)+\xi_{0}\right)\right) \mathrm{e}^{\mathrm{i}(-\kappa x+\omega t+\theta)},
\end{gathered}
$$

where Eqs. (19) and (20) represent dark soliton and singular soliton solutions respectively. These solitons are valid for

$$
a\left(a \kappa^{2}+\omega\right)<0 .
$$




$$
\begin{array}{r}
q(x, t)= \pm \sqrt{\frac{a \kappa^{2}+\omega}{2 b \kappa}} \\
\times \tan \left(\frac{1}{k} \sqrt{\frac{a \kappa^{2}+\omega}{2 a}}\left(k(x-v t)+\xi_{0}\right)\right) \mathrm{e}^{\mathrm{i}(-\kappa x+\omega t+\theta)}, \\
q(x, t)= \pm \sqrt{\frac{a \kappa^{2}+\omega}{2 b \kappa}} \\
\times \cot \left(\frac{1}{k} \sqrt{\frac{a \kappa^{2}+\omega}{2 a}}\left(k(x-v t)+\xi_{0}\right)\right) \mathrm{e}^{\mathrm{i}(-\kappa x+\omega t+\theta)},
\end{array}
$$

where Eqs.(21) and (22) represent singular periodic solutions. These solutions are valid for

$$
a\left(a \kappa^{2}+\omega\right)>0 \text {. }
$$

\subsubsection{Case-2}

By using transformation $U=V^{\frac{1}{2}}$, Eq. (8) becomes

$$
\begin{aligned}
& a k^{2}\left(-\left(V^{\prime}\right)^{2}+2 V V^{\prime \prime}\right)-4\left(a \kappa^{2}+\omega\right) V^{2} \\
& -8 b \kappa V^{3}=0 .
\end{aligned}
$$

Balancing $V V^{\prime \prime}$ or $\left(V^{\prime}\right)^{2}$ with $V^{3}$ in Eq. (23), then we get $N=2$. Consequently, we reach

$$
V(\xi)=a_{0}+a_{1}\left(\frac{\psi^{\prime}(\xi)}{\psi(\xi)}\right)+a_{2}\left(\frac{\psi^{\prime}(\xi)}{\psi(\xi)}\right)^{2} .
$$

Substituting Eq. (24) into Eq. (23) and then setting the coefficients of $\psi^{-j}, j=0,1,2,3,4$, 5 , 6 to zero, we obtain a set of algebraic equations as follows:

$\psi^{-6}$ coeff.:

$-8 a_{2}^{2}\left(\psi^{\prime}\right)^{6}\left(\kappa b a_{2}-a k^{2}\right)=0$,

$\psi^{-5}$ coeff.:

$-12 a_{2}\left(\psi^{\prime}\right)^{4}\left(\left(2 \kappa b a_{1} a_{2}-a k^{2} a_{1}\right) \psi^{\prime}+a k^{2} a_{2} \psi^{\prime \prime}\right)=0$,

$\psi^{-4}$ coeff.:

$-\left(\psi^{\prime}\right)^{3}\left[\left(4 \kappa^{2} a a_{2}^{2}+24 \kappa b a_{0} a_{2}^{2}+24 \kappa b a_{1}^{2} a_{2}\right.\right.$

$\left.-12 a k^{2} a_{0} a_{2}-3 a k^{2} a_{1}^{2}+4 \omega a_{2}^{2}\right) \psi^{\prime}+18 a k^{2} a_{1} a_{2} \psi^{\prime \prime}$

$\left.-4 a k^{2} a_{2}^{2} \psi^{\prime \prime \prime}\right]=0$,

$\psi^{-3}$ coeff.:

$$
\begin{aligned}
& -2\left(\psi^{\prime}\right)^{2}\left[\left(4 \kappa^{2} a a_{1} a_{2}+24 \kappa b a_{0} a_{1} a_{2}+4 \kappa b a_{1}^{3}\right.\right. \\
& \left.-2 a k^{2} a_{0} a_{1}+4 \omega a_{1} a_{2}\right) \psi^{\prime}+\left(10 a k^{2} a_{0} a_{2}+2 a k^{2} a_{1}^{2}\right) \psi^{\prime \prime} \\
& \left.-3 a k^{2} a_{1} a_{2} \psi^{\prime \prime \prime}\right]=0,
\end{aligned}
$$

$\psi^{-2}$ coeff.:

$$
\begin{gathered}
\left(-8 \kappa^{2} a a_{0} a_{2}-4 \kappa^{2} a a_{1}^{2}-24 \kappa b a_{0}^{2} a_{2}-24 \kappa b a_{0} a_{1}^{2}\right. \\
\left.-8 \omega a_{0} a_{2}-4 \omega a_{1}^{2}\right)\left(\psi^{\prime}\right)^{2}+\left(4 a k^{2} a_{0} a_{2}+2 a k^{2} a_{1}^{2}\right) \psi^{\prime} \psi^{\prime \prime \prime} \\
-6 a k^{2} a_{0} a_{1} \psi^{\prime} \psi^{\prime \prime}+\left(4 a k^{2} a_{0} a_{2}-a k^{2} a_{1}^{2}\right)\left(\psi^{\prime \prime}\right)^{2}=0,
\end{gathered}
$$

$\psi^{-1}$ coeff.:

$$
-2 a_{0} a_{1}\left[\left(4 \kappa^{2} a+12 \kappa b a_{0}+4 \omega\right) \psi^{\prime}-a k^{2} \psi^{\prime \prime \prime}\right]=0,
$$

$\psi^{0}$ coeff.:

$$
-4 a_{0}^{2}\left(\kappa^{2} a+2 \kappa b a_{0}+\omega\right)=0 .
$$

Solving this system, we obtain

$$
a_{0}=0, \quad a_{1}= \pm \frac{2 k \sqrt{a^{2} \kappa^{2}+a \omega}}{b \kappa}, \quad a_{2}=\frac{a k^{2}}{b \kappa},
$$

and

$$
\begin{aligned}
& \psi^{\prime \prime}= \pm \frac{2}{k} \sqrt{\frac{a \kappa^{2}+\omega}{a}} \psi^{\prime}, \\
& \psi^{\prime \prime \prime}=\frac{4\left(a \kappa^{2}+\omega\right)}{a k^{2}} \psi^{\prime} .
\end{aligned}
$$

From Eqs. (33) and (34), we can deduce that

$$
\psi^{\prime}= \pm \frac{k}{2} \sqrt{\frac{a}{a \kappa^{2}+\omega}} c_{1} \mathrm{e}^{ \pm \frac{2}{k} \sqrt{\frac{a \kappa^{2}+\omega}{a}} \xi},
$$

and

$$
\psi=\frac{a k^{2}}{4\left(a \kappa^{2}+\omega\right)} c_{1} \mathrm{e}^{ \pm \frac{2}{k} \sqrt{\frac{a \kappa^{2}+\omega}{a}} \xi}+c_{2},
$$

where $c_{1}$ and $c_{2}$ are constants of integration. Substituting Eq. (35) and (36) into Eq. (24), we obtain the following exact solution to Eq. (1):

$$
\begin{aligned}
& q(x, t)=\left[ \pm \frac{2 k \sqrt{a^{2} \kappa^{2}+a \omega}}{b \kappa}\right. \\
& \times\left(\frac{ \pm \frac{k}{2} \sqrt{\frac{a}{a \kappa^{2}+\omega}} c_{1} \mathrm{e}^{ \pm \frac{2}{k} \sqrt{\frac{a \kappa^{2}+\omega}{a}} k(x-v t)}}{\frac{a k^{2}}{4\left(a \kappa^{2}+\omega\right)} c_{1} \mathrm{e}^{ \pm \frac{2}{k} \sqrt{\frac{a \kappa^{2}+\omega}{a}} k(x-v t)}+c_{2}}\right) \\
& \left.+\frac{a k^{2}}{b \kappa}\left(\frac{ \pm \frac{k}{2} \sqrt{\frac{a}{a \kappa^{2}+\omega}} c_{1} \mathrm{e}^{ \pm \frac{2}{k} \sqrt{\frac{a \kappa^{2}+\omega}{a}} k(x-v t)}}{\frac{a k^{2}}{4\left(a \kappa^{2}+\omega\right)} c_{1} \mathrm{e}^{ \pm \frac{2}{k} \sqrt{\frac{a \kappa^{2}+\omega}{a}} k(x-v t)}+c_{2}}\right)^{2}\right]^{\frac{1}{2}} \\
& \times \mathrm{e}^{\mathrm{i}(-\kappa x+\omega t+\theta)} .
\end{aligned}
$$

If we set

$$
c_{1}=\frac{4\left(a \kappa^{2}+\omega\right)}{a k^{2}} \mathrm{e}^{ \pm \frac{2}{k} \sqrt{\frac{a \kappa^{2}+\omega}{a}} \xi_{0}}, \quad c_{2}= \pm 1,
$$

we obtain

$$
\begin{gathered}
q(x, t)=\left[-\frac{a \kappa^{2}+\omega}{b \kappa}\right. \\
\left.\times \operatorname{sech}^{2}\left(\frac{1}{k} \sqrt{\frac{a \kappa^{2}+\omega}{a}}\left(k(x-v t)+\xi_{0}\right)\right)\right]^{\frac{1}{2}} \mathrm{e}^{\mathrm{i}(-\kappa x+\omega t+\theta),} \\
q(x, t)=\left[\frac{a \kappa^{2}+\omega}{b \kappa}\right. \\
\left.\times \operatorname{csch}^{2}\left(\frac{1}{k} \sqrt{\frac{a \kappa^{2}+\omega}{a}}\left(k(x-v t)+\xi_{0}\right)\right)\right]^{\frac{1}{2}} \mathrm{e}^{\mathrm{i}(-\kappa x+\omega t+\theta)},
\end{gathered}
$$

where Eqs.(37) and (38) represent soliton and singular soliton solutions, respectively. These solitons are valid for

$$
a\left(a \kappa^{2}+\omega\right)>0 .
$$




$$
\begin{gathered}
q(x, t)=\left[-\frac{a \kappa^{2}+\omega}{b \kappa}\right. \\
\left.\times \sec ^{2}\left(\frac{1}{k} \sqrt{-\frac{a \kappa^{2}+\omega}{a}}\left(k(x-v t)+\xi_{0}\right)\right)\right]^{\frac{1}{2}} \mathrm{e}^{\mathrm{i}(-\kappa x+\omega t+\theta)} \\
q(x, t)=\left[-\frac{a \kappa^{2}+\omega}{b \kappa}\right. \\
\left.\times \csc ^{2}\left(\frac{1}{k} \sqrt{-\frac{a \kappa^{2}+\omega}{a}}\left(k(x-v t)+\xi_{0}\right)\right)\right]^{\frac{1}{2}} \mathrm{e}^{\mathrm{i}(-\kappa x+\omega t+\theta)}
\end{gathered}
$$

where Eqs.(39) and (40) represent singular periodic solutions. These solutions are valid for

$$
a\left(a \kappa^{2}+\omega\right)<0 \text {. }
$$

\section{Quick review of trial equation method}

In this section we outline the main steps of the trial equation method as following:

Step-1: Suppose a nonlinear partial differential equation (PDE) with time-dependent coefficients

$$
P\left(u, u_{t}, u_{x}, u_{t t}, u_{x t}, u_{x x}, \ldots\right)=0
$$

can be converted to an ODE

$$
Q\left(U, U^{\prime}, U^{\prime \prime}, U^{\prime \prime \prime}, \ldots\right)=0,
$$

using a travelling wave hypothesis $u(x, t)=U(\xi), \xi=$ $x-v t$, where $U=U(\xi)$ is an unknown function, $Q$ is a polynomial in the variable $U$ and its derivatives. If all terms contain derivatives, then Eq. (42) is integrated where integration constants are considered zeros.

Step-2: Take the trial equation

$$
\left(U^{\prime}\right)^{2}=F(U)=\sum_{l=0}^{N} a_{l} U^{l},
$$

where $a_{l},(l=0,1, \ldots, N)$ are constants to be determined. Substituting Eq. (43) and other derivative terms such as $U^{\prime \prime}$ or $U^{\prime \prime \prime}$ and so on into Eq. (42) yields a polynomial $G(U)$ of $U$. According to the balance principle we can determine the value of $N$. Setting the coefficients of $G(U)$ to zero, we get a system of algebraic equations. Solving this system, we can determine $v$ and values of $a_{0}, a_{1}, \ldots$, $a_{N}$. Step-3: Rewrite Eq. (43) by the integral form

$$
\pm\left(\xi-\xi_{0}\right)=\int \frac{\mathrm{d} U}{\sqrt{F(U)}} .
$$

According to the complete discrimination system of the polynomial, we classify the roots of $F(U)$, and solve the integral Eq. (44). Thus we obtain the exact solutions to Eq. (41).

\subsection{Application to $C-N L S E$}

This subsection will perform the trial equation method to derive soliton solutions to Eq. (1).

\subsubsection{Case-1}

Balancing $U^{\prime \prime}$ with $U^{3}$ in Eq. (8), then we get $N=4$. Using the solution procedure of the trial equation method, we obtain the system of algebraic equations as follows:
$U^{3}$ coeff.:

$a k^{2} a_{4}-b \kappa=0$,

$U^{2}$ coeff.:

$a k^{2} a_{3}=0$,

$U^{1}$ coeff.:

$a k^{2} a_{2}-\left(a \kappa^{2}+\omega\right)=0$,

$U^{0}$ coeff.:

$a k^{2} a_{1}=0$.

Solving the above system leads to

$$
\begin{aligned}
& a_{1}=0, \quad a_{2}=\frac{a \kappa^{2}+\omega}{a k^{2}}, \\
& a_{3}=0, \quad a_{4}=\frac{b \kappa}{a k^{2}} .
\end{aligned}
$$

Substituting these results into Eqs. (43) and (44), we get

$$
\pm\left(\xi-\xi_{0}\right)=\int \frac{\mathrm{d} U}{\sqrt{a_{0}+\frac{a \kappa^{2}+\omega}{a k^{2}} U^{2}+\frac{b \kappa}{a k^{2}} U^{4}}} .
$$

If we set $a_{0}=0$ in Eq. (49) and integrating with respect to $U$, we get the following exact solution of Eq. (1):

$$
\begin{aligned}
& q(x, t)= \pm \sqrt{-\frac{a \kappa^{2}+\omega}{b \kappa}} \\
& \times \operatorname{sech}\left(\sqrt{\frac{a \kappa^{2}+\omega}{a k^{2}}} k(x-v t)\right) \mathrm{e}^{\mathrm{i}(-\kappa x+\omega t+\theta),} \\
& q(x, t)= \pm \sqrt{\frac{a \kappa^{2}+\omega}{b \kappa}} \\
& \quad \times \operatorname{csch}\left(\sqrt{\frac{a \kappa^{2}+\omega}{a k^{2}}} k(x-v t)\right) \mathrm{e}^{\mathrm{i}(-\kappa x+\omega t+\theta)},
\end{aligned}
$$

where Eq. (50) and Eq. (51) represent solitons and singular soliton solutions, respectively. These solitons are valid for

$$
\begin{aligned}
& a\left(a \kappa^{2}+\omega\right)>0 \\
& q(x, t)= \pm \sqrt{-\frac{a \kappa^{2}+\omega}{b \kappa}} \\
& \quad \times \sec \left(\sqrt{-\frac{a \kappa^{2}+\omega}{a k^{2}}} k(x-v t)\right) \mathrm{e}^{\mathrm{i}(-\kappa x+\omega t+\theta),} \\
& q(x, t)= \pm \sqrt{-\frac{a \kappa^{2}+\omega}{b \kappa}} \\
& \quad \times \csc \left(\sqrt{-\frac{a \kappa^{2}+\omega}{a k^{2}}} k(x-v t)\right) \mathrm{e}^{\mathrm{i}(-\kappa x+\omega t+\theta),}
\end{aligned}
$$

where Eq. (52) and Eq. (53) represent singular periodic solutions. These solutions are valid for

$$
a\left(a \kappa^{2}+\omega\right)<0 .
$$

If we set $a_{0}=\frac{\left(a \kappa^{2}+\omega\right)^{2}}{4 a k^{2} b \kappa}$ in Eq. (49) and integrating with respect to $U$, we get the following exact solution of Eq. (1): 


$$
\begin{aligned}
& q(x, t)= \pm \sqrt{\frac{a \kappa^{2}+\omega}{2 b \kappa}} \\
& \times \tan \left(\sqrt{\frac{a \kappa^{2}+\omega}{2 a k^{2}}} k(x-v t)\right) \mathrm{e}^{\mathrm{i}(-\kappa x+\omega t+\theta),} \\
& q(x, t)= \pm \sqrt{\frac{a \kappa^{2}+\omega}{2 b \kappa}} \\
& \times \cot \left(\sqrt{\frac{a \kappa^{2}+\omega}{2 a k^{2}}} k(x-v t)\right) \mathrm{e}^{\mathrm{i}(-\kappa x+\omega t+\theta)},
\end{aligned}
$$

where Eq. (54) and Eq. (55) represent singular periodic solutions. These solutions are valid for

$$
\begin{aligned}
& a\left(a \kappa^{2}+\omega\right)>0 \\
& q(x, t)= \pm \sqrt{-\frac{a \kappa^{2}+\omega}{2 b \kappa}} \\
& \quad \times \tanh \left(\sqrt{-\frac{a \kappa^{2}+\omega}{2 a k^{2}}} k(x-v t)\right) \mathrm{e}^{\mathrm{i}(-\kappa x+\omega t+\theta)} \\
& q(x, t)= \pm \sqrt{-\frac{a \kappa^{2}+\omega}{2 b \kappa}} \\
& \quad \times \operatorname{coth}\left(\sqrt{-\frac{a \kappa^{2}+\omega}{2 a k^{2}}} k(x-v t)\right) \mathrm{e}^{\mathrm{i}(-\kappa x+\omega t+\theta)}
\end{aligned}
$$

where Eq. (56) and Eq. (57) represent dark and singular soliton solutions, respectively. These solitons are valid for

$$
a\left(a \kappa^{2}+\omega\right)<0 \text {. }
$$

\subsubsection{Case-2}

By using transformation $U=V^{\frac{1}{2}}$, Eq. (8) becomes

$$
\begin{aligned}
& a k^{2}\left(-\left(V^{\prime}\right)^{2}+2 V V^{\prime \prime}\right)-4\left(a \kappa^{2}+\omega\right) V^{2} \\
& -8 b \kappa V^{3}=0 .
\end{aligned}
$$

Balancing $V V^{\prime \prime}$ or $\left(V^{\prime}\right)^{2}$ with $V^{3}$ in Eq. (58), then we get $N=3$. Using the solution procedure of the trial equation method, we obtain the system of algebraic equations as follows:

$$
\begin{aligned}
& V^{3} \text { coeff.: } \\
& a k^{2} a_{3}-4 b \kappa=0, \\
& V^{2} \text { coeff.: } \\
& a k^{2} a_{2}-4\left(a \kappa^{2}+\omega\right)=0, \\
& V^{0} \text { coeff.: } \\
& a k^{2} a_{0}=0 .
\end{aligned}
$$

Solving the above system leads to

$$
a_{0}=0, \quad a_{2}=\frac{4\left(a \kappa^{2}+\omega\right)}{a k^{2}}, \quad a_{3}=\frac{4 b \kappa}{a k^{2}} .
$$

Substituting these results into Eqs. (43) and (44), we get

$$
\pm\left(\xi-\xi_{0}\right)=\int \frac{\mathrm{d} V}{\sqrt{a_{1} V+\frac{4\left(a \kappa^{2}+\omega\right)}{a k^{2}} V^{2}+\frac{4 b \kappa}{a k^{2}} V^{3}}} .
$$

If we set $a_{1}=0$ in Eq. (62) and integrating with respect to $V$, we get the following exact solution of Eq. (1):

$$
\begin{aligned}
& q(x, t)= \pm \sqrt{-\frac{a \kappa^{2}+\omega}{b \kappa}} \\
& \times \operatorname{sech}\left(\sqrt{\left.\frac{a \kappa^{2}+\omega}{a k^{2}} k(x-v t)\right) \mathrm{e}^{\mathrm{i}(-\kappa x+\omega t+\theta)},}\right. \\
& q(x, t)= \pm \sqrt{\frac{a \kappa^{2}+\omega}{b \kappa}} \\
& \times \operatorname{csch}\left(\sqrt{\frac{a \kappa^{2}+\omega}{a k^{2}}} k(x-v t)\right) \mathrm{e}^{\mathrm{i}(-\kappa x+\omega t+\theta)},
\end{aligned}
$$

where Eq. (63) and Eq. (64) represent bright and singular soliton solutions, respectively. These solitons are valid for

$$
\begin{aligned}
& a\left(a \kappa^{2}+\omega\right)>0 . \\
& q(x, t)= \pm \sqrt{\frac{a \kappa^{2}+\omega}{b \kappa}} \\
& \quad \times \sec \left(\sqrt{-\frac{a \kappa^{2}+\omega}{a k^{2}}} k(x-v t)\right) \mathrm{e}^{\mathrm{i}(-\kappa x+\omega t+\theta),} \\
& q(x, t)= \pm \sqrt{\frac{a \kappa^{2}+\omega}{b \kappa}} \\
& \quad \times \csc \left(\sqrt{-\frac{a \kappa^{2}+\omega}{a k^{2}}} k(x-v t)\right) \mathrm{e}^{\mathrm{i}(-\kappa x+\omega t+\theta),}
\end{aligned}
$$

where Eq. (52) and Eq. (53) represent singular periodic solutions. These solutions are valid for

$$
a\left(a \kappa^{2}+\omega\right)<0 .
$$

\section{Conclusions}

In this paper, soliton solutions are studied for C-NLSE with the Bohm potential. Solitons and shock waves are obtained by the modified simple equation method and trial equation method along with necessary constraint conditions that guarantees the existence of such solitons. As a byproduct, singular periodic solutions are revealed as a by-product of these approaches and these are also listed. The results of this paper reveal yet another couple of powerful mathematical techniques to retrieve soliton solutions in the context of nuclear physics.

\section{Future work}

The results of this paper are indeed encouraging and thus lead to several additional avenues of research in this field. Later, the model will be addressed with fractional temporal evolution as well as time-dependent coefficients along with generalized version of C-NLSE. Additional integration schemes will enlighten the model further along. Some of these schemes that can shed more light into this 
model are extended trial function method, Kudryashov's algorithm, the Lie symmetry analysis and many more. Numerically, this model can be made to glow from a visual perspective. Such research activities are all under way and their results will be visible, with time, across a variety of journals.

\section{Acknowledgments}

The work of the fourth author (Q.Z.) was supported by the National Science Foundation for Young Scientists of Wuhan Donghu University. The fifth author (S.P.M.) would like to thank the research support provided by the Department of Mathematics and Statistics at Tshwane University of Technology and the support from the South African National Foundation under Grant Number 92052 IRF1202210126. The research work of sixth author (M.R.B.) was supported by the grant number NPRP 8-028-1-001 from QNRF and is thankful for it.

\section{References}

[1] U. Agiletti, L. Griguolo, R. Jackiw, S.Y. Pi, D. Semirara, Phys. Rev. Lett. 77, 4406 (1996).

[2] A. Biswas, Nucl. Phys. B 806, 457 (2009).
[3] A. Biswas, Int. J. Theor. Phys. 48, 3403 (2009).

[4] A. Biswas, Int. J. Theor. Phys. 49, 79 (2010).

[5] A. Biswas, D. Milovic, Phys. At. Nucl. 74, 755 (2011).

[6] A. Biswas, A.H. Kara, E. Zerrad, Open Nucl. Part. Phys. J. 4, 21 (2011).

[7] G. Ebadi, A. Yildirim, A. Biswas, Roman. Rep. Phys. 64, 357 (2012)

[8] M.S. Ismail, K.S. Al-Basyouni, A. Aydin, Boundary Value Problems 2015, 9 (2015).

[9] R. Jackiw, S.Y. Pi, Phys. Rev. Lett. 64, 2969 (1990).

[10] R. Jackiw, S.Y. Pi, Phys. Rev. D 44, 2524 (1991).

[11] R. Jackiw, J. Nonlin. Math. Phys. 4, 261 (1997).

[12] A.G. Johnpillai, A. Yildirim, A. Biswas, Roman. J. Phys. 57, 545 (2012).

[13] J.H. Lee, C.K. Lin, O.K. Pashaev, Chaos Solitons Fractals 19, 109 (2004).

[14] M. Mirzazadeh, M. Eslami, A. Biswas, Acta Phys. Pol. B 45, 845 (2014).

[15] A. Nishino, Y. Umeno, M. Wadati, Chaos Solitons Fractals 9, 1063 (1998). 